\title{
有界外乱のもとでの不確かなシステムの
}

ミニマックス推定

一回帰ベクトルが周期的でない場合*

\section{Minimax Estimation of Uncertain Systems in the Presence of Bounded Disturbance} - Nonperiodic Regressor Case*

Wataru KITAMURA $^{\dagger}$ and Yasumasa FUJISAKI ${ }^{\ddagger}$

\section{1.はじめに}

有界外乱のもとでの不確かなシステムの同定におい て，システムパラメータの公称值だけでなく，外乱とパ ラメータ不確かさの大きさの上界も推定する方法 [1] が 提案されている。これは，有界外乱のみが存在するとき のミニマックス推定 [2] をパラメータの不確かさも存在 する場合へと拡張したものであり，ある確率的仮定のも とで，推定誤差が 0 に収束することが示されている。 た だし，その解析では，回帰ベクトルに周期性を仮定して おり，この仮定がないときの議論はされていない.

本論文では, 回帰べクトルの周期性を仮定せずに，推 定誤差を解析する。具体的には，大きさが一定の 2 組 の回帰ベクトルが持続的励振条件を満たし，外乱とパラ メー夕不確かさが最悪值近傍をとる確率が 0 でないと仮 定し，推定誤差の上界を信頼度付きで求める，得られる 結果より，これらの条件のもとでは，たとえ回帰べクト ルが周期的でなくても, サンプル数を増加させれば推定 誤差が 0 に収束することがわかる。

\section{2. ミニマックス推定}

対象システムは, 1 入出力の離散時間線形システム

$$
y_{i}=\phi_{i}^{\mathrm{T}}\left(\theta+\eta_{i}\right)+v_{i}
$$

であるとする。ここで, 添字 $i$ は時刻, $y_{i} \in \mathbf{R}$ はシステ 厶の出力, $\phi_{i} \in \mathbf{R}^{m}$ は回帰ベクトル, $\theta \in \mathbf{R}^{m}$ は同定す ベきシステムパラメータの公称值である. また, $v_{i} \in \mathbf{R}$ は外乱, $\eta_{i} \in \mathbf{R}^{m}$ はパラメータの不確かさであり,

$$
\left|v_{i}\right| \leq \epsilon, \quad\left\|\eta_{i}\right\|_{2} \leq \delta
$$

* 原稿受付 2004 年 12 月 13 日

$\dagger$ 神戸大学 大学院 自然科学研究科 Graduate School of Science and Technology, Kobe University; 1-1 Rokkodai, Nada, Kobe, Hyogo 657-8501, JAPAN

‡神戸大学 工学部 情報知能工学科 Department of Computer and Systems Engineering, Kobe University; 1-1 Rokkodai, Nada, Kobe, Hyogo 657-8501, JAPAN Key Words: minimax estimation, uncertain systems, bounded disturbance, nonperiodic regressor.
とする。ただし，上界 $\epsilon$ や $\delta$ は未知であるとする。な お, $\|\bullet\|_{2}$ は $l_{2}$ ノルム $\left\|\eta_{i}\right\|_{2} \doteq\left(\eta_{i}^{\mathrm{T}} \eta_{i}\right)^{1 / 2}$ とする.

回帰ベクトルの大きさが, $\left\|\phi_{i}^{(1)}\right\|_{2}=b_{1},\left\|\phi_{i}^{(2)}\right\|_{2}=b_{2}$ と規格化された 2 組の入出力デー夕 $\left\{y_{i}^{(1)}, \phi_{i}^{(1)}\right\}, i=$ $1,2, \ldots, N_{1},\left\{y_{i}^{(2)}, \phi_{i}^{(2)}\right\}, i=1,2, \ldots, N_{2}$ が与えられてい るとする。ただし $0<b_{1}<b_{2}<\infty$ である.このとき， $\theta$ の推定值 $\widehat{\theta}$ は, 各組ごとにミニマックス推定を用いると,

$$
\widehat{\theta}_{j} \doteq \arg \min _{\hat{\theta}} \max _{i}\left|y_{i}^{(j)}-\left(\phi_{i}^{(j)}\right)^{\mathrm{T}} \hat{\theta}\right|, \quad j=1,2
$$

と求まる $[1]$. また，このときの出力誤差の最大值を

$$
\xi_{j} \doteq \max _{i}\left|y_{i}^{(j)}-\left(\phi_{i}^{(j)}\right)^{\mathrm{T}} \widehat{\theta}_{j}\right|, \quad j=1,2
$$

と定義すれば, $\epsilon, \delta$ の推定值 $\widehat{\epsilon}, \widehat{\delta}$ は

$$
\widehat{\epsilon} \doteq \frac{b_{2} \xi_{1}-b_{1} \xi_{2}}{b_{2}-b_{1}}, \quad \widehat{\delta} \doteq \frac{\xi_{2}-\xi_{1}}{b_{2}-b_{1}}
$$

と求まる $[1]$.

\section{3. 推定誤差の確率的評価}

ある $n>0, \alpha>0, \beta>0$ が存在し，任意の $i_{0}, 1 \leq$ $i_{0} \leq N-n+1$ に対して

$$
\alpha^{2} I \leq \sum_{i=i_{0}}^{i_{0}+n-1} \phi_{i} \phi_{i}{ }^{\mathrm{T}} \leq \beta^{2} I
$$

が成り立つとき，回帰ベクトル $\phi_{i}, i=1,2, \ldots, N$ は持続 的励振 (Persistently Exciting, PE) 条件を満たすとい う [3]. ここで, $A \leq B$ は, $B-A$ が準正定であることを 意味する。いま， $\mathbf{N}$ は自然数の集合， $\mathcal{N}(\bullet)$ は集合・の 要素数をあらわすとすれば, 以下の補題が成り立つ [4].

【補題 1】回帰ベクトル $\phi_{i}, i=1,2, \ldots, N$ は $\mathrm{PE}$ 条件を満足し， $N$ は $2 n$ の整数倍であると仮定して $N=2 n r$ とする。ただし,$r \in \mathbf{N}$ である。また， $q \doteq$ $\min _{p}\{p \in \mathbf{N}: p \alpha \geq \pi \beta \sqrt{n(m-1)}\}$ を用いて $\mathcal{P} \doteq 2+$ $2 q(q-1)^{m-2}$ と定義する。 このとき，(i) から (iii) を満 たす時刻列の部分列 $\left\{i_{k}\right\}$ の集合 $\mathcal{K}$ が存在する.

(i) $\mathcal{N}\left(\left\{i_{k}\right\}\right) \geq r, \quad \forall\left\{i_{k}\right\} \in \mathcal{K}$ 
(ii) $\mathcal{N}(\mathcal{K}) \leq \mathcal{P}$

(iii) 任意の $x \in \mathbf{R}^{m}$ に対して, ある $\left\{i_{k}\right\}$ が存在して

$$
\begin{aligned}
x^{\mathrm{T}} \phi_{i_{k}} & \leq-\frac{\alpha}{2 \sqrt{n}}\|x\|_{2}, \quad \forall k \\
x^{\mathrm{T}} \phi_{i_{k}} & \geq \frac{\alpha}{2 \sqrt{n}}\|x\|_{2}, \quad \forall k
\end{aligned}
$$

のいずれかが成り立つ.

外乱 $v_{i}$ が $\left|v_{i}\right| \leq \epsilon$ を満たす確率変数であり, 任意の $\rho>0, i$ について, ある $p_{v}(\rho)>0$ が存在し

$$
\begin{aligned}
& \operatorname{Pr}\left\{-\epsilon \leq v_{i} \leq-(\epsilon-\rho)\right\} \geq p_{v}(\rho) \\
& \operatorname{Pr}\left\{\epsilon-\rho \leq v_{i} \leq \epsilon\right\} \geq p_{v}(\rho)
\end{aligned}
$$

が成り立つとき， $\epsilon$ は密着性 (tightness) をもつという [5]. 同様に, パラメータ不確かさ $\eta_{i}$ が $\left\|\eta_{i}\right\|_{2} \leq \delta$ を満たす 確率変数であり，任意の $\mu>0, i, \phi_{i}$ について，ある $p_{\eta}(\mu)>0$ が存在し

$$
\begin{aligned}
& \operatorname{Pr}\left\{-\delta\left\|\phi_{i}\right\|_{2} \leq \phi_{i}{ }^{\mathrm{T}} \eta_{i} \leq-(\delta-\mu)\left\|\phi_{i}\right\|_{2}\right\} \geq p_{\eta}(\mu) \\
& \operatorname{Pr}\left\{(\delta-\mu)\left\|\phi_{i}\right\|_{2} \leq \phi_{i}{ }^{\mathrm{T}} \eta_{i} \leq \delta\left\|\phi_{i}\right\|_{2}\right\} \geq p_{\eta}(\mu)
\end{aligned}
$$

が成り立つとき, $\delta$ は密着性をもつという [6].ここで, $\operatorname{Pr}\{\bullet\}$ は事象・が起こる確率をあらわす，このとき，次 の補題 [6]が成り立つ.

【補題 2】外乱 $v_{i}$, パラメー夕の不確かさ $\eta_{i}$ が独立 な確率変数であり, 上界 $\epsilon$ と $\delta$ がともに密着性をもて ば，任意の $\rho>0, \mu>0, i, \phi_{i}$ について

$$
\begin{gathered}
\operatorname{Pr}\left\{-\left(\epsilon+\delta\left\|\phi_{i}\right\|_{2}\right) \leq v_{i}+\phi_{i}{ }^{\mathrm{T}} \eta_{i}\right. \\
\left.\quad \leq-(\epsilon-\rho)-(\delta-\mu)\left\|\phi_{i}\right\|_{2}\right\} \geq p_{v}(\rho) p_{\eta}(\mu) \\
\operatorname{Pr}\left\{\epsilon-\rho+(\delta-\mu)\left\|\phi_{i}\right\|_{2} \leq v_{i}+\phi_{i}{ }^{\mathrm{T}} \eta_{i}\right. \\
\left.\leq \epsilon+\delta\left\|\phi_{i}\right\|_{2}\right\} \geq p_{v}(\rho) p_{\eta}(\mu)
\end{gathered}
$$

が成立する。

2 組の回帰ベクトル $\phi_{i}^{(1)}, \phi_{i}^{(2)}$ がそれぞれ PE 条件を 満足するとし，それに対応してPE条件と補題 1 に扔 る定数 $\alpha, n, r, \mathcal{P}$ の右下に添字 $j=1,2$ を付けてあら わすとすれば，以下の定理が成り立つ。

【定理 1】(1)式のシステムに执いて, $\phi_{i}^{(j)}, j=1,2$ が確定的, $\mathrm{PE}$ 条件を満たすとする。 また, $v_{i}, \eta_{i}$ が独 立な確率変数であり, それらの上界 $\epsilon, \delta$ が密着性をも つとする.このとき, $\theta$ の推定誤差について,

$$
\operatorname{Pr}\left\{\left\|\theta-\widehat{\theta}_{j}\right\| \leq \frac{2 \sqrt{n_{j}}}{\alpha_{j}}\left(\rho+b_{j} \mu\right)\right\} \geq c_{p_{j}}
$$

が成り立つ.ここで, $c_{p_{j}}$ は

$$
c_{p_{j}} \leq 1-\mathcal{P}_{j}\left(1-p_{v}(\rho) p_{\eta}(\mu)\right)^{r_{j}}
$$

を満たす正の定数である.

（証明）証明の手順は， $j=1,2$ とも同様なので, 以下 では, $j$ は省略する。まず,

$$
e_{i} \doteq v_{i}+\phi_{i}{ }^{\mathrm{T}} \eta_{i}, \quad \nu \doteq \epsilon+\delta b
$$

とすれば，(2) 式より， $\nu \pm e_{i} \geq 0$ である.さて，ミニ マックス推定が (3) 式で抗こなわれることに注意すれば,

$$
\left|y_{i}-\phi_{i}^{\mathrm{T}} \widehat{\theta}\right| \leq \nu
$$

であることがわかる．よって，(1)式，(12)式より

$$
-\left(\nu+e_{i}\right) \leq \phi_{i}{ }^{\mathrm{T}}(\theta-\widehat{\theta}) \leq \nu-e_{i}
$$

である。いま, $x=\theta-\widehat{\theta}$ とおけば，補題 1 の (iii) より, (6) 式または $(7)$ 式のどちらかを満たす $\left\{i_{k}\right\} \in \mathcal{K}$ が必ず 存在するので,

$$
\|\theta-\widehat{\theta}\|_{2} \leq \frac{2 \sqrt{n}}{\alpha} \max _{\left\{i_{k}\right\} \in \mathcal{K}} \psi\left(\left\{i_{k}\right\}\right)
$$

と評価できる。ここで, $\psi\left(\left\{i_{k}\right\}\right)$ は

$$
\psi\left(\left\{i_{k}\right\}\right) \doteq \begin{cases}\min _{k}\left(\nu+e_{i_{k}}\right) & \text { if }(6) \text { holds } \\ \min _{k}\left(\nu-e_{i_{k}}\right) & \text { if }(7) \text { holds }\end{cases}
$$

である。したがって推定誤差は

$$
\begin{aligned}
& \operatorname{Pr}\left\{\|\theta-\widehat{\theta}\|_{2} \leq \frac{2 \sqrt{n}}{\alpha}(\rho+\mu b)\right\} \\
& \geq \operatorname{Pr}\left\{\max _{\left\{i_{k}\right\} \in \mathcal{K}} \psi\left(\left\{i_{k}\right\}\right) \leq \rho+\mu b\right\} \\
& =1-\operatorname{Pr}\left\{\max _{\left\{i_{k}\right\} \in \mathcal{K}} \psi\left(\left\{i_{k}\right\}\right)>\rho+\mu b\right\}
\end{aligned}
$$

と確率的に評価できる. いま $\psi\left(\left\{i_{k}\right\}\right)=\min _{k}\left(\nu+e_{i_{k}}\right)$ の とき $\psi\left(\left\{i_{k}\right\}\right)$ が最大值をとる場合には, 補題 $\mathbf{1}$ の (ii), 補題 1 の(i), 補題 2 の (8) 式を順に適用することにより

$$
\begin{aligned}
& \operatorname{Pr}\left\{\max _{\left\{i_{k}\right\} \in \mathcal{K}} \min _{k}\left(\nu+e_{i_{k}}\right)>\rho+\mu b\right\} \\
& \leq \mathcal{N}(\mathcal{K}) \cdot \operatorname{Pr}\left\{\min _{k}\left(\nu+e_{i_{k}}\right)>\rho+\mu b\right\} \\
& \leq \mathcal{P} \cdot \operatorname{Pr}\left\{\nu+e_{i_{k}}>\rho+\mu b \forall k\right\} \\
& \leq \mathcal{P}\left(1-\operatorname{Pr}\left\{\nu+e_{i} \leq \rho+\mu b\right\}\right)^{r} \\
& \leq \mathcal{P}\left(1-p_{v}(\rho) p_{\eta}(\mu)\right)^{r}
\end{aligned}
$$

となる.一方, $\psi\left(\left\{i_{k}\right\}\right)=\min _{k}\left(\nu-e_{i_{k}}\right)$ のとき $\psi\left(\left\{i_{k}\right\}\right)$ が最大值をとる場合にも, 同様に

$$
\begin{aligned}
& \operatorname{Pr}\left\{\max _{\left\{i_{k}\right\} \in \mathcal{K}} \min _{k}\left(\nu-e_{i_{k}}\right)>\rho+\mu b\right\} \\
& \leq \mathcal{P}\left(1-\operatorname{Pr}\left\{\nu-e_{i} \leq \rho+\mu b\right\}\right)^{r} \\
& \leq \mathcal{P}\left(1-p_{v}(\rho) p_{\eta}(\mu)\right)^{r}
\end{aligned}
$$

となる.これら $(14) \sim(16)$ 式より (10) 式を得る.

つぎに, $\epsilon$ と $\delta$ の推定誤差について考察する.

【補題 3】定理 1 と同様の仮定をおく。このとき，

$$
\operatorname{Pr}\left\{\epsilon-2 \rho+(\delta-2 \mu) b_{j} \leq \xi_{j} \leq \epsilon+\delta b_{j}\right\} \geq c_{p_{j}}
$$

が成立する。

（証明）定理 1 の証明と同様に，jを省略する。な お，(12) 式より，(4) 式の $\xi$ の上界に関する不等号は 
確率 1 で成立するので， $\xi$ の下界のみ議論する。まず, $\phi_{i}{ }^{\mathrm{T}}(\theta-\widehat{\theta}) \leq 0$ のとき，(11) 式で定義した $e_{i}, \nu$ につ いて

$$
-\nu \leq e_{i} \leq-\nu+\rho+\mu b
$$

であると仮定すれば，(13) 式から

$$
-(\rho+\mu b) \leq \phi_{i}^{\mathrm{T}}(\theta-\widehat{\theta}) \leq 0
$$

となり，

$$
\left|e_{i}\right|-\left|\phi_{i}^{\mathrm{T}}(\theta-\widehat{\theta})\right| \geq \nu-2(\rho+\mu b)
$$

を得る。一方， $\phi_{i}^{\mathrm{T}}(\theta-\widehat{\theta})>0$ のとき，

$$
\nu-(\rho+\mu b) \leq e_{i} \leq \nu
$$

であると仮定すれば，(13)式から

$$
0<\phi_{i}^{\mathrm{T}}(\theta-\widehat{\theta}) \leq \rho+\mu b
$$

となり，同様に (19) 式が成立する，以上より，補題 2 を 用いて (18), (20) 式が成り立つ確率を評価すれば，結局

$$
\begin{aligned}
& \operatorname{Pr}\left\{\left|e_{i}\right|-\left|\phi_{i}^{\mathrm{T}}(\theta-\widehat{\theta})\right| \geq \nu-2(\rho+\mu b)\right\} \\
& \geq p_{v}(\rho) p_{\eta}(\mu)
\end{aligned}
$$

であることがわかる。ささて $\xi$ の下界の確率は

$$
\begin{aligned}
& \operatorname{Pr}\{\xi \geq \epsilon-2 \rho+(\delta-2 \mu) b\} \\
& =1-\operatorname{Pr}\{\xi<\nu-2(\rho+\mu b)\}
\end{aligned}
$$

と書ける。これに, 補題 1 の (ii), 補題 1 の (i), (21) 式 を順に適用することにより

$$
\begin{aligned}
& \operatorname{Pr}\{\xi<\nu-2(\rho+\mu b)\} \\
& \leq \operatorname{Pr}\left\{\max _{\left\{i_{k}\right\} \in \mathcal{K}}\left\{\max _{k}\left|y_{i_{k}}-\phi_{i_{k}} \mathrm{~T} \widehat{\theta}\right|\right\}<\nu-2(\rho+\mu b)\right\} \\
& \leq \mathcal{N}(\mathcal{K}) \cdot \operatorname{Pr}\left\{\max _{k}\left|y_{i_{k}}-\phi_{i_{k}}{ }^{\mathrm{T}} \widehat{\theta}\right|<\nu-2(\rho+\mu b)\right\} \\
& \leq \mathcal{P} \cdot \operatorname{Pr}\left\{\left|e_{i_{k}}\right|-\left|\phi_{i_{k}}{ }^{\mathrm{T}}(\theta-\widehat{\theta})\right|<\nu-2(\rho+\mu b), \forall k\right\} \\
& =\mathcal{P}\left(1-\operatorname{Pr}\left\{\left|e_{i}\right|-\left|\phi_{i}^{\mathrm{T}}(\theta-\widehat{\theta})\right| \geq \nu-2(\rho+\mu b)\right\}\right)^{r} \\
& \leq \mathcal{P}\left(1-p_{v}(\rho) p_{\eta}(\mu)\right)^{r}
\end{aligned}
$$

となる。これら $(22),(23)$ 式より (17) 式を得る.

このとき, 以下の定理が成り立つ.

【定理 2】(1)式のシステムにおいて, $\phi_{i}^{(j)}, j=1,2$ が確定的で， PE 条件を満足するとする．また， $v_{i}$ と $\eta_{i}$ が独立な確率変数であり， $\epsilon ， \delta$ が密着性をもつとする. このとき, $\epsilon, \delta$ の推定誤差について以下が成り立つ.

$$
\begin{gathered}
\operatorname{Pr}\left\{-\frac{2 b_{1}\left(\rho+b_{2} \mu\right)}{b_{2}-b_{1}} \leq \epsilon-\widehat{\epsilon} \leq \frac{2 b_{2}\left(\rho+b_{1} \mu\right)}{b_{2}-b_{1}}\right\} \\
\operatorname{Pr}\left\{-\frac{2\left(\rho+b_{1} \mu\right)}{b_{p_{1}} c_{p_{2}}-b_{1}} \leq \delta-\widehat{\delta} \leq \frac{2\left(\rho+b_{2} \mu\right)}{b_{2}-b_{1}}\right\} \\
\geq c_{p_{1}} c_{p_{2}}
\end{gathered}
$$

（証明）推定值 $\widehat{\epsilon}, \widehat{\delta}$ の定義である (5) 式を, 補題 3 の $\xi_{j}$ に関する $(17)$ 式の不等式を用いて評価すれば,

$$
\begin{aligned}
\widehat{\epsilon} & \geq \frac{b_{2}\left\{\epsilon-2 \rho+(\delta-2 \mu) b_{1}\right\}-b_{1}\left(\epsilon+\delta b_{2}\right)}{b_{2}-b_{1}} \\
& =\epsilon-\frac{2 b_{2}\left(\rho+b_{1} \mu\right)}{b_{2}-b_{1}} \\
\widehat{\epsilon} & \leq \frac{b_{2}\left(\epsilon+\delta b_{1}\right)-b_{1}\left\{\epsilon-2 \rho+(\delta-2 \mu) b_{2}\right\}}{b_{2}-b_{1}} \\
& =\epsilon+\frac{2 b_{1}\left(\rho+b_{2} \mu\right)}{b_{2}-b_{1}} \\
\widehat{\delta} & \geq \frac{\epsilon-2 \rho+(\delta-2 \mu) b_{2}-\left(\epsilon+\delta b_{1}\right)}{b_{2}-b_{1}} \\
& =\delta-\frac{2\left(\rho+b_{2} \mu\right)}{b_{2}-b_{1}} \\
\widehat{\delta} & \leq \frac{\left\{\epsilon+\rho+(\delta+\mu) b_{2}\right\}-\left\{\epsilon-2 \rho+(\delta-2 \mu) b_{1}\right\}}{b_{2}-b_{1}} \\
& =\delta+\frac{2\left(\rho+b_{1} \mu\right)}{b_{2}-b_{1}}
\end{aligned}
$$

となり，(24), (25) 式の不等式を得る.これらの信頼度 は, 補題 $\mathbf{3}$ の評価が $j=1,2$ でともに成り立つ確率とな り，これらは互いに独立であるから， $c_{p_{1}} c_{p_{2}}$ となる.

定理 1, 2 より，推定誤差が $\rho, \mu$ に対して線形であ $\eta$, その信頼度 $c_{p_{j}}$ が $r_{j} \rightarrow \infty$ で 1 に漸近することが わかる. よって, 参考文献 [1] と同様の手順で, サンプ ル数の増加につれて推定誤差が 0 に確率収束することを 示すことができる.つまり, 回㷌べクトルの周期性を仮 定しない場合でも，仮定した場合 [1] と同様に，推定誤 差の収束性を保証できる.

\section{参 考文 献}

[1] 北村, 藤崎: 有界外乱のもとでの不確かなシステムのミニ マックス推定; 計測自動制御学会論文集, Vol. 41, No. 2, pp. 111-117 (2005)

[2] E.-W. Bai, H. Cho and R. Tempo: Convergence properties of the membership set; Automatica, Vol. 34, No. 10, pp. 1245-1249 (1998)

[3] S. Dasgupta and Y.-F. Huang: Asymptotically convergent modified recursive least squares with data dependent updating and forgetting factor for system with bounded noise; IEEE Trans. on Information Theory, Vol. 33, No. 3, pp. 383-392 (1987)

[4] H. Akçay: The size of the membership set in a probabilistic framework; Automatica, Vol. 40, No. 2, pp. 253-260 (2004)

[5] E.-W. Bai, R. Tempo and H. Cho: Membership set estimators; Size, optimal input, complexity and relations with least squares; IEEE Trans. on Circuits and Systems -Part I, Vol. 42, No. 5, pp. 266-277 (1995)

[6] W. Kitamura and Y. Fujisaki: Convergence properties of the membership set in the presence of disturbance and parameter uncertainty; 計測自動制御学会論文集, Vol. 39, No. 4, pp. 382-387 (2003) 